\title{
Pensamiento social y posicionamiento político: representaciones sociales de la derecha y la izquierda en Colombia
}

Social thought and political positioning: social representations of the right and the left in Colombia

Pensamento social e posicionamento político: representações sociais da direita e da esquerda na Colômbia

\section{Georgie Alexánder Echeverri Vásquez ${ }^{1}$ Álvaro Rafael Santana Peixoto ${ }^{2}$ Ana Raquel Rosas Torres ${ }^{3}$}

Recibido: 17 de agosto de 2020 Aprobado: 6 de marzo de 2021 Publicado: 5 de mayo de 2021

Cómo citar este artículo: Echeverri Vásquez, G.A., Santana Peixoto, A.R. y Rosas Torres, A.R. (2021). Pensamiento social y posicionamiento político: representaciones sociales de la derecha y la izquierda en Colombia. Pensando Psicología, 17(1), 1-24. doi: https://doi.org/10.16925/2382-3984.2021.01.01

Artículo de investigación. https://doi.org/10.16925/2382-3984.2021.01.01

1 Universidade do Estado do Rio de Janeiro - UERJ

Correo electrónico: mensajerodevida@gmail.com

ORCID: https://orcid.org/0000-0002-5253-0738

2 Universidade Federal do Espírito Santo-UFES

Correo electrónico: alvarorafael.peixoto@gmail.com

ORCID: https://orcid.org/0000-0002-1480-7919

3 Universidade Federal da Paraíba-UFPB

Correo electrónico: arr.torres@gmail.com

ORCID: https://orcid.org/0000-0002-3161-0309 


\title{
Resumen
}

Introducción: las representaciones sociales son teorías del sentido común sobre un objeto polémico, construidas consensualmente por los grupos. Desde el punto de vista de la epistemología interaccional, se trata de formas de pensamiento social o esquemas de interpretación del mundo directamente asociados con las prácticas sociales, es decir, a la forma en que los grupos se comportan.

Objetivos: 1) identificar la posible representación social de la 'derecha' y la 'izquierda' en Colombia. 2) establecer la relación entre el efecto movilizador del rótulo y la adhesión o rechazo a postulados de ambas vertientes ideológicas.

Método: se aplicó un cuestionario a 104 colombianos adultos, segmentados de acuerdo con su orientación ideológica de derecha, izquierda o centro. El cuestionario contenía una técnica de asociación libre de palabras (TALP), a partir de la cual se realizó un análisis prototípico y un análisis de similitud; y una escala Likert, con base en la cual se efectuó un análisis de varianza (ANOVA).

Resultados: se identificó una posible representación social de la 'derecha' estructurada en torno de la figura del expresidente Álvaro Uribe y dos posibles representaciones de la 'izquierda', una vinculada al comunismo y otra a las expectativas por la consolidación del proceso de paz. Con base en la teoría de la identidad social también fue posible evidenciar en los votantes de izquierda el efecto movilizador del rótulo como activador de una representación social vinculada a la identidad grupal.

Palabras clave: representación social, identidad social, redes sociales digitales, rótulo, Colombia

\begin{abstract}
Introduction: Social representations are common sense theories about a controversial object, which is constructed consensually by groups. From the point of view of interactional epistemology, social representations are forms of social thought or schemes for interpreting the world directly associated to social practices, that is, to the way groups behave.
\end{abstract}

Objectives: 1) To identify the possible social representation of the 'right' and the 'left' in Colombia. 2) To establish the relationship between the mobilizing effect of the label and the adherence or rejection of postulates of both ideological strands.

Method: A questionnaire was applied to 104 Colombian adults, segmented according to their ideological orientation of right, left or center. The questionnaire contained a word-free association technique, from which a prototypical analysis and a similarity analysis were performed; and a Likert scale, based on which an ANOVA test was carried out.

Results: A possible social representation of the 'right' structured around former president Álvaro Uribe and two possible representations of the 'left' were identified, one linked to communism and the other to expectations for the consolidation of the peace process. Based on the theory of social identity, it was also possible to show in left voters the mobilizing effect of the label as a trigger of a social representation linked to group identity.

Keywords: social representation, social identity, social media, label, Colombia.

\section{Resumo}

Introdução: As representações sociais são teorias do senso comum sobre um objeto polêmico construídas consensualmente pelos grupos. Do ponto de vista da epistemologia interacional, trata-se de formas do pensamento social ou esquemas de interpretação do mundo diretamente associados às práticas sociais, quer dizer, à forma como os grupos se comportam. 
Objetivos: 1) Identificar a possível representação social da 'direita' e da 'esquerda' na Colômbia. 2) Estabelecer a relação entre o efeito mobilizador do rótulo e a adesão ou rejeição de postulados advindos de ambas a vertentes ideológicas.

Método: Aplicou-se um questionário em 104 colombianos adultos, segmentados de acordo com a sua orientação ideológica de direita, esquerda ou centro. 0 questionário continha uma técnica de associação livre de palavras (TALP), a partir da qual foi realizada uma análise prototípica e uma análise de similitude; e uma escala Likert, como base para a realização de uma análise de variância (ANOVA).

Resultados: Identificou-se uma possível representação social da 'direita' estruturada em torno da figura do ex-presidente Álvaro Uribe e duas possíveis representações da 'esquerda', uma associada ao comunismo e mais outra ligada às expectativas pela consolidação do processo de paz. Embasado na teoria da identidade social também foi possível evidenciar nos eleitores de esquerda o efeito mobilizador do rótulo como ativador de uma representação social vinculada à identidade grupal.

Palavras-chave: representação social, identidade social, redes sociais digitais, rótulo, Colômbia.

En este artículo nos proponemos, a partir de un estudio psicosocial realizado con 104 participantes colombianos, identificar y analizar la posible representación social de la 'derecha' y la 'izquierda' en Colombia, utilizando la técnica de asociación libre de palabras (TALP) como base para la realización de los análisis prototípico y de similitud, comúnmente usados en el abordaje estructural de las representaciones sociales. Asimismo, pretendemos establecer la posible relación entre el efecto movilizador del rótulo y la adhesión o rechazo a los postulados de ambas vertientes ideológicas, por medio de un análisis de varianza (ANOVA).

Uno de los hechos que ha marcado la historia reciente de Colombia ha sido, sin lugar a dudas, la firma del acuerdo de paz entre el gobierno del entonces presidente Juan Manuel Santos y la guerrilla de las Fuerzas Armadas Revolucionarias de Colombia (FARC). Luego de tres años y ocho meses de negociaciones, el Gobierno colombiano y las FARC presentaron, el 24 de agosto de 2016, un documento llamado "Acuerdo general para la terminación del conflicto y la construcción de una paz estable y duradera" (Alto Comisionado para la Paz de Colombia, 2016). Los medios de información internacional registraron el hecho como la posibilidad real que tenían los colombianos de pasar la página de una guerra (Casey, 2016; Gaymard, 2016).

Según cifras divulgadas por el Centro Nacional de Memoria Histórica (CNMH, 2016), en el periodo comprendido entre 1958 y 2012, el conflicto en Colombia dejó cerca de 220000 personas asesinadas por los actores armados - guerrillas, paramilitares y fuerzas del Estado-, así como 25000 personas desaparecidas y 4,7 millones de desplazados.

En el marco de ese proceso de paz hubo un hecho que llamó poderosamente la atención: el plebiscito del 2 de octubre de 2016, en el que el 50,21\% de los votantes 
4 Pensamiento social y posicionamiento político: representaciones sociales de la derecha y la izquierda en Colombia

rechazaron el acuerdo suscrito, frente al 49,78\% que lo respaldaron. La pregunta obligada, entonces, fue: ¿qué había llevado a los colombianos a rechazar un acuerdo que, en principio, significaba el fin de la guerra con la guerrilla más antigua de América Latina?

Estudios desarrollados en el campo de la Ciencia Política y del Derecho han presentado diferentes hipótesis: 1) asocian la procedencia geográfica de los votos con la intensidad territorial del conflicto (Basset, 2018); 2) muestran que los ciudadanos afectados por la violencia insurgente y contrainsurgente asumieron una posición más acomodaticia y no guerrerista, frente a otros ciudadanos que, al no padecer las consecuencias de la violencia, optaron por socavar la paz en las urnas (Pachenkina y Gamboa, 2019); y 3) resaltan el papel de las emociones, especialmente, el miedo, en el proceso de la campaña electoral, tanto por parte de los promotores del Sí como del No (Gómez, 2017; Cardona y Londoño, 2018; Echeverri y Andrade, 2019);

Lo cierto es que el resultado del plebiscito de 2016, que llevó a una revisión de los acuerdos (Botero, 2017), también incidió en la forma en que se desarrolló la campaña presidencial de 2018, especialmente, si tenemos en cuenta que los dos candidatos con posibilidades reales de llegar al Palacio de Nariño representaban dos extremos ideológicos: Iván Duque, candidato del Partido Centro Democrático, cuyo jefe político es el expresidente y senador Álvaro Uribe Vélez, el más férreo opositor a los acuerdos de paz y líder natural de la campaña del No; y Gustavo Petro, exalcalde de Bogotá y quien fuera miembro del movimiento guerrillero $\mathbf{M}$-19, desmovilizado en la década de 90 luego de aquel proceso de paz.

En el campo de las redes sociales digitales comenzó a intensificarse el debate, sobre todo entre quienes apoyaban a Duque, partidario de una revisión de los acuerdos suscritos en La Habana, y los seguidores de Petro, defensor de dichos acuerdos. Lo interesante de ese debate digital, que motivó la realización del presente estudio, fue la alusión recurrente al rótulo "ser de derecha" o "ser de izquierda".

Si analizamos la historia política de Colombia, desde la segunda mitad del siglo XIX, cuando comienza el proceso de consolidación ideológica del Partido Liberal y el Partido Conservador, hasta llegar a la primera mitad del siglo XX, cuando se transforman en partidos de masas, es posible evidenciar, dos proyectos políticos antagónicos, aparentemente irreconciliables.

Los liberales consideraban injusto el orden tradicional y estaban dispuestos a apoyar a los grupos populares en sus conflictos con propietarios y empresarios reaccionarios, y a quitar a la Iglesia su poder temporal. Y los conservadores veían a los liberales como demagogos dispuestos a pro- 
mover la lucha de clases y la destrucción del orden, mediante la violencia y la expropiación injusta de los bienes legítimos de los propietarios. (Melo, 2017, p. 201)

Esa dinámica política de dicotomización del mundo social (Rehm, 2014), exacerbada por la bipolaridad propia de la Guerra Fría, no solo legitimaba el discurso de odio contra el opositor, considerado como enemigo, sino también el uso de la violencia física como instrumento político para exterminarlo. Como señala Melo (2017), el régimen de partido único, sin oposición, de 1958 a 1978, conocido como el Frente Nacional o alternancia en el poder de los partidos Liberal y Conservador, "eliminó los odios de los partidos, pero al mismo tiempo destruyó su papel como promotores de un proyecto social" (p. 322), convirtiéndolos en redes clientelistas.

Ahora bien, el debate digital entre Duque y Petro parecía no obedecer a tesis partidarias, históricamente consolidadas - de hecho, ninguno de ellos pertenece a los dos partidos históricos tradicionales-, sino a candidatos que representaban ideas contrarias sobre el funcionamiento del Estado y de la economía, y que llevaban a sus seguidores/detractores a identificarse con ese ideario y a promoverlo en sus redes sociales digitales, de manera tal que era posible diferenciar un "nosotros" y un "ellos", partiendo de los rótulos "izquierda" y "derecha".

Fue con base en la observación de ese fenómeno, que decidimos realizar una lectura psicosocial del mismo, partiendo de la premisa según la cual es posible estudiar la realidad mediante una perspectiva integradora, que articule niveles psicológicos y sociológicos de análisis (Álvaro y Garrido, 2006). En ese orden de ideas, optamos por apoyarnos en el desarrollo de los conceptos de representación social e identidad social con el propósito de identificar: 1) la posible representación social de los conceptos 'izquierda' y 'derecha' construida y compartida por los participantes del estudio y 2) el efecto movilizador del rótulo o etiqueta, asociado a la imagen de los candidatos Iván Duque y Gustavo Petro.

\section{Representaciones sociales y procesos identitarios}

El concepto de representación social surge en el marco de lo que se conoce en la Psicología Social como epistemología interaccional o psicosociología del conocimiento (Moscovici, 1961; Marková, 2006; Sá, 2015; Castorina, 2016). Se trata de formas de conocimiento de un objeto, construidas colectivamente por un grupo, que 
poseen un sentido eminentemente práctico. En otras palabras, se pueden definir como esquemas de interpretación de la realidad -Moscovici (1961), su precursor, las denominaba teorías del sentido común- que están directamente relacionados con la pertenencia a un grupo y con la posición que dicho grupo ocupa en la sociedad (Jodelet, 1989).

Entendidas como formas del pensamiento social, las representaciones sociales se materializan en las conversaciones cotidianas (Rouquette, 1998). Como explica Sá (1998), para que exista una representación social, dichos temas u objetos deben: 1) ser relevantes para el grupo, 2) generar un conocimiento estructurado, 3) favorecer la formulación de juicios, 4) estar asociados con las prácticas sociales y 5) cumplir una función de diferenciación intergrupal.

Es importante anotar que, en el marco de lo que teóricamente se ha denominado "arquitectura del pensamiento social" (Rouquette, 1998; Echeverri, Wolter y Peixoto, 2019), existe una relación jerárquica de generación cognitiva, que permite identificar la coexistencia de diferentes formas de pensamiento social, a saber, las ideologías, las representaciones sociales, las actitudes y las opiniones. Dicha organización jerárquica se rige por los principios de la estabilidad y de la integración, que funcionan a modo de secuencia; es decir, se puede pasar de la estabilidad extrema y generalidad de una ideología a la inestabilidad total y especificidad de una opinión. Esta acotación es necesaria para efectos de comprensión de este estudio porque permite entender que las representaciones sociales no son opiniones, sino teorías ingenuas o del sentido común más estables, que los grupos construyen y sedimentan históricamente alrededor de objetos específicos.

Dada la importancia que tienen las representaciones sociales en el proceso de relación de un grupo con su entorno, es fundamental tener en cuenta que una de sus funciones es precisamente la formación de la identidad grupal (Abric, 1994); en otras palabras, las representaciones sociales están relacionadas tanto con el sentido de pertenencia a un grupo como con los procesos de diferenciación intergrupal. En línea con esto, Tajfel $(1978,1981)$ establece que la identidad social está asociada a la noción de pertenencia a los grupos sociales y al significado emocional y valorativo de esa pertenencia. El famoso experimento de los "grupos mínimos" (Tajfel y Turner, 1986) Ilevó a los autores a proponer que ese significado emocional y valorativo se explicaba por el favoritismo que el endogrupo experimentaba hacia sí mismo (grupo de pertenencia), en detrimento del otro grupo (exogrupo). Hablando desde el punto de vista psicosociológico, y siguiendo esa línea de investigación marcada por la Escuela de Bristol, desarrollada posteriormente por la Escuela de Ginebra (Deschamps, 1983; 
Doise, 1987, 1988), es posible afirmar que la identidad social integra tanto las condiciones objetivas de la relación intergrupos como la dimensión cognitiva.

Existen dos procesos esenciales en la formación de esa identidad social o grupal, que ocurren en el marco de la interacción entre un grupo observador -endogrupo- y un grupo externo observado -exogrupo-: se trata de la categorización social y la comparación social. Las representaciones sociales actúan en ambos procesos, permitiéndole al endogrupo evaluarse a sí mismo y también al exogrupo con el propósito de considerar las posibilidades de cooperación o conflicto con él, así como establecer parámetros de valoración o subestimación de ese exogrupo (Amâncio, 2004; Cabecinhas, 2007).

Deschamps y Moliner (2004) afirman que existen representaciones sociales específicas que actúan en la construcción de la identidad de un grupo, tales como la representación que el grupo tiene de sí mismo, de los otros grupos y de la estructura social a la que pertenecen. Esas representaciones sociales intergrupos, además de producir juicios y prácticas, contribuyen a que los grupos se perciban en el entramado social y propendan por la búsqueda de una identidad positiva.

Cuando un grupo se autocategoriza y categoriza a los grupos de su entorno, se crean esquemas de simplificación y generalización, que guían las acciones y los conceptos sobre los sujetos que pertenecen a esos grupos; la comparación social, por su parte, ocurre cuando el endogrupo afirma su propia identidad y se distancia de las otras, partiendo de la representación social que tiene del exogrupo (Marques, 1988).

Ahora bien, los procesos de categorización y comparación social no solo se orientan hacia la identidad social, sino que también generan efectos colaterales como el estereotipo, que suprime la identidad individual de un sujeto en virtud de su grupo de pertenencia. Los sujetos también se atribuyen a sí mismos características del grupo de pertenencia, proceso denominado autoestereotipia, en el que prima la valoración de la propia identidad social en detrimento de las características individuales (Marques y Páez, 2004).

En ese orden de ideas, en una situación de conflicto, el exogrupo representa una amenaza para el endogrupo, lo que favorece el surgimiento de prejuicios, entendidos como actitudes adversas u hostiles hacia una persona, por el simple hecho de pertenecer a un grupo (Fiske, 1998). Así como cualquier proceso electoral favorece el surgimiento de estereotipos negativos, también permite que aumenten las demostraciones de prejuicio en sus diversos niveles, y ese es el foco del estudio que presentaremos a continuación. 
8 Pensamiento social y posicionamiento político: representaciones sociales de la derecha y la izquierda en Colombia

\section{Método}

\section{Participantes}

El estudio contó con 104 participantes colombianos, 54 mujeres y 50 hombres, con un promedio de edad de 42,5 años ( $\mathrm{DE}=9,48)$, pertenecientes al estrato socioeconómico $3(D E=1,25)$ en una escala de 1 a 6, como se utiliza habitualmente en Colombia, siendo 1 el estrato bajo-bajo y 6 el estrato alto (Uribe-Mallarino, 2008). De los 104 participantes, 77 tenían estudios superiores completos con especialización o maestría. Con respecto a la localización geográfica, 66 participantes afirmaron vivir en Medellín y ocho en Bogotá, los mayores centros urbanos de Colombia; los 30 restantes reportaron su residencia en ciudades intermedias o fuera del país.

\section{Procedimiento}

El estudio se desarrolló en dos momentos. El primero de ellos, que denominamos mapeamiento digital, consistió en hacerle un seguimiento a la línea del tiempo (timeline) de las páginas de Facebook de los 104 participantes con el propósito de identificar su posicionamiento político un mes antes de la primera vuelta de las elecciones presidenciales de 2018 en Colombia, que se llevó a cabo el 27 de mayo de ese año.

Una vez realizadas esas primeras votaciones, en las que resultaron electos los candidatos Iván Duque Márquez, del partido Centro Democrático y Gustavo Petro Urrego, del partido Colombia Humana, invitamos formalmente a esos 104 colombianos a participar en la investigación, cuyo segundo momento se desarrolló en un lapso de tres semanas, previas a la segunda vuelta, que tuvo lugar el 17 de junio de 2018.

Para efectos de organización de la lógica interna del estudio, los 104 participantes fueron clasificados en tres grupos de acuerdo con el mapeamiento digital inicial: 21 participantes, que mostraron abiertamente su apoyo al candidato Gustavo Petro, fueron clasificados como 'votantes de izquierda'; 22 participantes que manifestaron su adhesión pública a Iván Duque fueron clasificados como 'votantes de derecha'; y los 61 participantes restantes, que habían apoyado al candidato Sergio Fajardo -la tercera opción con mayores posibilidades de pasar a la segunda vuelta- o que no habían respaldado públicamente las campañas de Gustavo Petro o Iván Duque, fueron clasificados como 'votantes de centro'.

En ese segundo momento se elaboraron tres cuestionarios digitales diferentes en la plataforma Google Forms, para que cada una de las personas vinculadas a las tres categorías arriba mencionadas los diligenciase. El abordaje de cada uno de los 104 participantes fue personalizado, a través de un mensaje dirigido al buzón de 
Facebook de cada uno de ellos, y se realizó en el periodo de mayor polarización de las campañas políticas, especialmente en el campo de las redes sociales digitales.

Teniendo en cuenta que las representaciones sociales desempeñan un papel fundamental en la formación de la identidad social, así como en el surgimiento de estereotipos y prejuicios, consideramos pertinente identificar, de una forma exploratoria, elementos relacionados con la representación social que los participantes tenían sobre la derecha y la izquierda en Colombia.

Por esa razón, la primera parte del cuestionario consistió en una técnica de asociación libre de palabras (TALP), formulada en los siguientes términos: "Actualmente, en los medios de comunicación de Colombia y en las redes sociales, se hace referencia a candidatos de derecha o candidatos de izquierda. ¿Cuáles son las tres primeras palabras o expresiones que pasan por su cabeza cuando lee o escucha la palabra 'derecha'?". El mismo ejercicio de evocaciones libres se hizo con la palabra 'izquierda'. Esta técnica, formulada en el cuadro del abordaje estructural de la teoría de las representaciones sociales (Vergès, 1994; Oliveira et al, 2005, Wolter, 2018) se propone identificar las ideas o cognemas que, eventualmente, podrían hacer parte del núcleo central y del sistema periférico de una representación socialmente construida.

La segunda parte del cuestionario abordó aspectos actitudinales con el propósito de evidenciar si el posicionamiento (aceptación o rechazo) ante una premisa por parte de un grupo podría estar relacionado con la presencia o ausencia de un rótulo, en este caso, imagénico, es decir, asociado a la imagen del candidato que la enunciaba.

Como se muestra en la tabla 1, construimos siete afirmaciones a partir de la lectura de los programas de gobierno de los candidatos Iván Duque y Gustavo Petro, relacionadas con temas centrales en cualquier campaña electoral, redactadas de forma tal que fuera posible atribuírselas a uno u otro candidato, y les pedimos a los participantes que, utilizando una escala Likert de cinco puntos, manifestasen si estaban desde 'totalmente de acuerdo' o 'totalmente en desacuerdo' con ellas.

Tabla 1. Afirmaciones de la escala Likert presentadas a los participantes

\begin{tabular}{lll}
\hline \multicolumn{2}{c}{ Tema } & \multicolumn{1}{c}{ Premisas generales de un eventual programa de gobierno } \\
\hline 1 & Infancia & $\begin{array}{l}\text { La alimentación balanceada de los niños es un elemento fundamental en la aten- } \\
\text { ción integral a la primera infancia. }\end{array}$ \\
\hline 2 & Educación & $\begin{array}{l}\text { A través de la jornada escolar única se garantiza la inclusión y el derecho a la } \\
\text { educación }\end{array}$ \\
\hline 3 & Investigación & $\begin{array}{l}\text { Incrementar el presupuesto para investigación científica y tecnológica permite } \\
\text { fortalecer el sector productivo }\end{array}$ \\
\hline
\end{tabular}


(viene)

\begin{tabular}{lll}
\hline \multicolumn{1}{c}{ Tema } & \multicolumn{1}{c}{ Premisas generales de un eventual programa de gobierno } \\
\hline 4 & Emprendimiento & $\begin{array}{l}\text { El estímulo al emprendimiento es una estrategia que contribuye a la formalización } \\
\text { del empleo }\end{array}$ \\
\hline 5 & $\begin{array}{l}\text { Reactivación del } \\
\text { campo }\end{array}$ & La creación de cadenas productivas y asociativas favorece la reactivación del campo. \\
\hline 6 & Equidad de género & $\begin{array}{l}\text { La promoción de iniciativas de equidad de género contribuye a corregir la } \\
\text { discriminación contra la mujer }\end{array}$ \\
\hline 7 & Medio ambiente & $\begin{array}{l}\text { La protección del agua es un eje fundamental de la política de sostenibilidad } \\
\text { ambiental }\end{array}$ \\
\hline
\end{tabular}

Fuente: elaboración propia

Ahora bien, la presentación de las afirmaciones tenía una particularidad. Como muestra la tabla 2, a la mitad de los votantes, tanto de derecha como de izquierda, dichas premisas se les presentaron acompañadas de la fotografía de Iván Duque (la imagen oficial de la campaña), explicándoles que se trataba de afırmaciones extraídas de su programa de gobierno, y con la otra mitad de los votantes, tanto de izquierda como de derecha, se procedió de forma semejante, pero utilizando la fotografía de Gustavo Petro. A la mitad de los votantes de centro, por su parte, se les presentaron las mismas afirmaciones, sin fotografía, aduciendo que se trataba de premisas de 'un partido de derecha' y a la otra mitad, también sin fotografía, se le dijo que se trataba de afirmaciones propias de 'un partido de izquierda'.

Tabla 2. Distribución de los participantes según el tipo de rótulo

\begin{tabular}{lcccc}
\hline Participantes & $\begin{array}{c}\text { Fotografía } \\
\text { Iván Duque }\end{array}$ & $\begin{array}{c}\text { Fotografía } \\
\text { Gustavo Petro }\end{array}$ & $\begin{array}{c}\text { Partido } \\
\text { de derecha }\end{array}$ & $\begin{array}{c}\text { Partido } \\
\text { de izquierda }\end{array}$ \\
\hline Derecha & 12 & 10 & & \\
\hline Izquierda & 10 & 11 & 34 & 27 \\
\hline Centro & & & & 61 \\
\hline Total & $\mathbf{2 2}$ & $\mathbf{2 1}$ & & \\
\hline
\end{tabular}

Fuente: elaboración propia.

\section{Análisis de los datos}

Los datos obtenidos en la técnica de asociación libre de palabras (TALP), luego de su proceso de lematización, fueron sometidos a un análisis prototípico y a un análisis de similitud por medio del programa Iramuteq (Interface de R pour les Analyses Multidimensionnelles de Textes et de Questionnaires). El análisis prototípico (Oliveira 
et al, 2005; Wachelke y Wolter, 2011) tiene en cuenta tanto la frecuencia con que aparece un cognema como su promedio de evocación. En ese orden de ideas, el criterio para hacer parte del posible núcleo central de una representación social es que el cognema tenga una frecuencia alta y un promedio de evocación bajo. El análisis de similitud, por su parte, permite identificar gráficamente las relaciones entre los cognemas por medio de la aplicación de índices probabilísticos (Real y Vargas, 1996), sugiriendo pistas que complementan el análisis prototípico.

Los datos provenientes de la escala Likert fueron llevados a un análisis de varianza (ANOVA), con el objetivo de determinar si existía una diferencia significativa al comparar las medias (Dancey y Reidy, 2006). Una vez constatada la significancia, se procedió a aplicar el Test HSD de Tukey para identificar exactamente las premisas en las que se presentaban dichas diferencias significativas.

\section{Resultados}

La técnica de asociación libre de palabras (TALP) permitió recolectar 151 evocaciones relacionadas con el término inductor 'derecha' y 153 palabras o expresiones asociadas al término inductor 'izquierda'. Es importante destacar que, para efectos de dicho ejercicio, se tuvo en cuenta el universo total de participantes (104), teniendo en cuenta que todos eran colombianos y potenciales votantes.

Tabla 3. Análisis prototípico del término inductor 'derecha'

\begin{tabular}{|c|c|c|c|c|c|c|}
\hline & \multicolumn{3}{|c|}{ Promedio de evocación $<=1.85$} & \multicolumn{3}{|c|}{ Promedio de evocación >1,85 } \\
\hline & Cognema & Frecuencia & Promedio & Cognema & Frecuencia & Promedio \\
\hline \multirow{3}{*}{$\begin{array}{l}\text { Frecuencia } \\
<8,41\end{array}$} & Conservador & 20 & 1,4 & Corrupción & 21 & 2 \\
\hline & Tradición & 16 & 1,8 & Uribe & 17 & 2,1 \\
\hline & Continuidad & 10 & 1,6 & Capitalismo & 9 & 1,9 \\
\hline \multirow{6}{*}{$\begin{array}{l}\text { Frecuencia } \\
>=8,41\end{array}$} & Democracia & 5 & 1,8 & $\begin{array}{l}\text { Lo mismo de } \\
\text { siempre }\end{array}$ & 8 & 2 \\
\hline & Polarización & 4 & 1,2 & Paramilitarismo & 8 & 2,2 \\
\hline & Extremismo & 4 & 1,8 & Poder & 5 & 2,2 \\
\hline & Falsos positivos & 4 & 1,8 & Maquinaria & 3 & 3 \\
\hline & Igualdad & 3 & 1,3 & Impunidad & 3 & 2,3 \\
\hline & Orden & 3 & 1,7 & & & \\
\hline
\end{tabular}

Fuente: elaboración propia. 
La tabla 3, correspondiente a la representación social de la derecha, nos muestra un posible núcleo central, es decir, un conjunto de cognemas más estables compartidos por el grupo de votantes potenciales, compuesto por tres ideas con mayor frecuencia y menor promedio de evocación (cuadrante superior izquierdo). Dichas ideas son: conservador, tradición y continuidad.

Vale la pena destacar, igualmente, dos cognemas que aparecen en lo que técnicamente se denomina primera periferia (cuadrante superior derecho), es decir, elementos que hacen parte de la representación social, pero al ser evocados con menor rapidez, no aparecen como candidatos al núcleo central: en este caso, nos estamos refiriendo a corrupción y Uribe, que obtuvieron frecuencias incluso más altas que las de los cognemas que integrarían el núcleo central.

Ahora bien, al someter los resultados del análisis prototípico a un análisis de similitud (figura 1), podemos confirmar, desde el punto de vista gráfico -colores lila, verde, aguamarina y rosado-, la formación de campos semánticos o de significación alrededor de las ideas más frecuentemente citadas en la TALP, que el análisis prototípico nos presentó como posibles cognemas del núcleo central de la representación social de la derecha y que el programa Iramuteq resalta en los círculos más grandes.

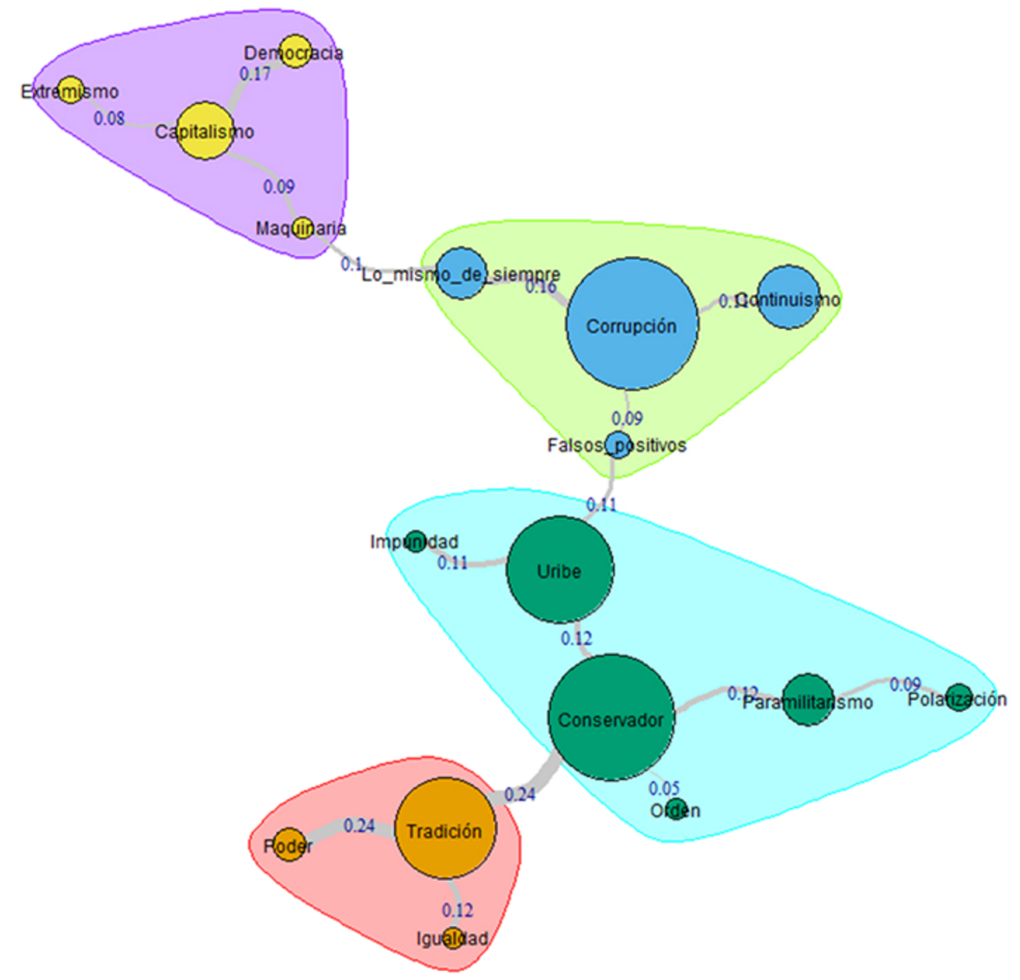

Figura 1. Análisis de similitud del término inductor 'derecha' Fuente: elaboración propia. 
En el primer campo semántico, se destaca la fuerte relación que los participantes atribuyeron a los conceptos capitalismo y democracia. Un segundo campo semántico, asociado al anterior por medio del cognema maquinarias (redes de clientelismo político), se forma alrededor de la corrupción como elemento central, conectado con ideas que denotan desaprobación y rechazo, tales como lo mismo de siempre y continuismo.

Asociada a ese segundo campo de significaciones aparece la figura del expresidente Uribe y el cognema conservador, como polos centrales de escándalos como los falsos positivos (asesinatos de civiles por parte de militares ocurridos durante su gobierno y denunciadas tanto por organizaciones defensoras de Derechos Humanos como por los medios de comunicación en Colombia) y el paramilitarismo. Finalmente, se evidencian los mayores índices de conexión $(0,24)$ entre los cognemas conservador, tradición y poder, que forman el cuarto y último campo semántico producto del análisis de similitud correspondiente a la representación socialmente construida de la derecha en Colombia.

Tabla 4. Análisis prototípico del término inductor 'izquierda'

\begin{tabular}{|c|c|c|c|c|c|c|}
\hline & \multicolumn{3}{|c|}{ Promedio de evocación $<=1,92$} & \multicolumn{3}{|c|}{ Promedio de evocación >1,92 } \\
\hline & Cognema & Frecuencia & Promedio & Cognema & Frecuencia & Promedio \\
\hline \multirow{5}{*}{$\begin{array}{l}\text { Frecuencia } \\
<6,32\end{array}$} & Cambio & 18 & 1,7 & Guerrilla & 14 & 2,2 \\
\hline & Comunismo & 14 & 1,5 & Socialismo & 14 & 2 \\
\hline & Revolución & 12 & 1,6 & Equidad & 9 & 2,1 \\
\hline & & & & Opción & 7 & 2,4 \\
\hline & & & & Oportunidad & 7 & 2,1 \\
\hline \multirow{9}{*}{$\begin{array}{l}\text { Frecuencia } \\
>=6,32\end{array}$} & $\begin{array}{l}\text { Castrocha- } \\
\text { vismo }\end{array}$ & 5 & 1,4 & Venezuela & 6 & 2 \\
\hline & Petro & 4 & 1 & Esperanza & 5 & 2 \\
\hline & Libertad & 4 & 1,5 & Expropiación & 4 & 2,5 \\
\hline & Inclusión & 3 & 1,7 & Pobreza & 4 & 2,8 \\
\hline & $\begin{array}{l}\text { Igualdad } \\
\text { social }\end{array}$ & 3 & 1,3 & Anarquismo & 4 & 3 \\
\hline & Ateísmo & 3 & 1,7 & Populismo & 3 & 2 \\
\hline & Alternativa & 3 & 1,7 & Liberal & 3 & 2,3 \\
\hline & Dictadura & 3 & 1 & $\begin{array}{l}\text { Derechos } \\
\text { Humanos }\end{array}$ & 2 & 2,7 \\
\hline & & & & $\begin{array}{l}\text { Proceso de } \\
\text { paz }\end{array}$ & 3 & 2,7 \\
\hline
\end{tabular}

Fuente: elaboración propia. 
Con respecto al análisis prototípico del término inductor "izquierda", que aparece en la tabla 4, los cognemas cambio, comunismo y revolución aparecen en el cuadrante correspondiente al posible núcleo central, al presentar una frecuencia alta y un promedio de evocación bajo. Como sucedió en el análisis prototípico del término inductor "derecha", en la primera periferia también aparecieron dos ideas con una frecuencia alta (guerrilla y socialismo).

Como muestra la figura 2, las relaciones entre cognemas permitieron la formación de cuatro campos semánticos, tres de ellos conectados directamente con la idea de comunismo como punto central del rizoma. El primer campo presenta una asociación fuerte $(0,33)$ del comunismo con el socialismo y, a partir de este, con elementos como revolución, igualdad social, inclusión y Derechos Humanos. El segundo campo conecta el comunismo con el cognema guerrilla, siendo esta una idea frecuentemente evocada, pero sin conexión con otros elementos. El tercer campo semántico, por su parte, asocia el comunismo con la crisis política y económica de Venezuela, apareciendo ideas como castrochavismo (neologismo difundido inicialmente en Colombia por el expresidente Álvaro Uribe), expropiación, Petro y pobreza.

A diferencia del análisis de similitud del término inductor 'derecha', en el que los cuatro campos de significación se encontraban interconectados, en el de la 'izquierda' aparece un campo de significación independiente, a partir del cual es posible inferir: 1) la centralidad del cognema opción asociado a cambio -idea frecuentemente evocada- así como a alternativa, oportunidad y proceso de paz; y 2) una fuerte conexión (la mayor de todas: 0,33) entre oportunidad y esperanza. La aparente independencia de ese campo semántico sugiere la posible configuración de otra representación social de la izquierda, compartida por un subgrupo dentro del universo de los participantes, hipótesis que podría comprobarse aplicando otras técnicas propias del abordaje estructural de las representaciones sociales que escapan al objetivo exploratorio del presente estudio. 


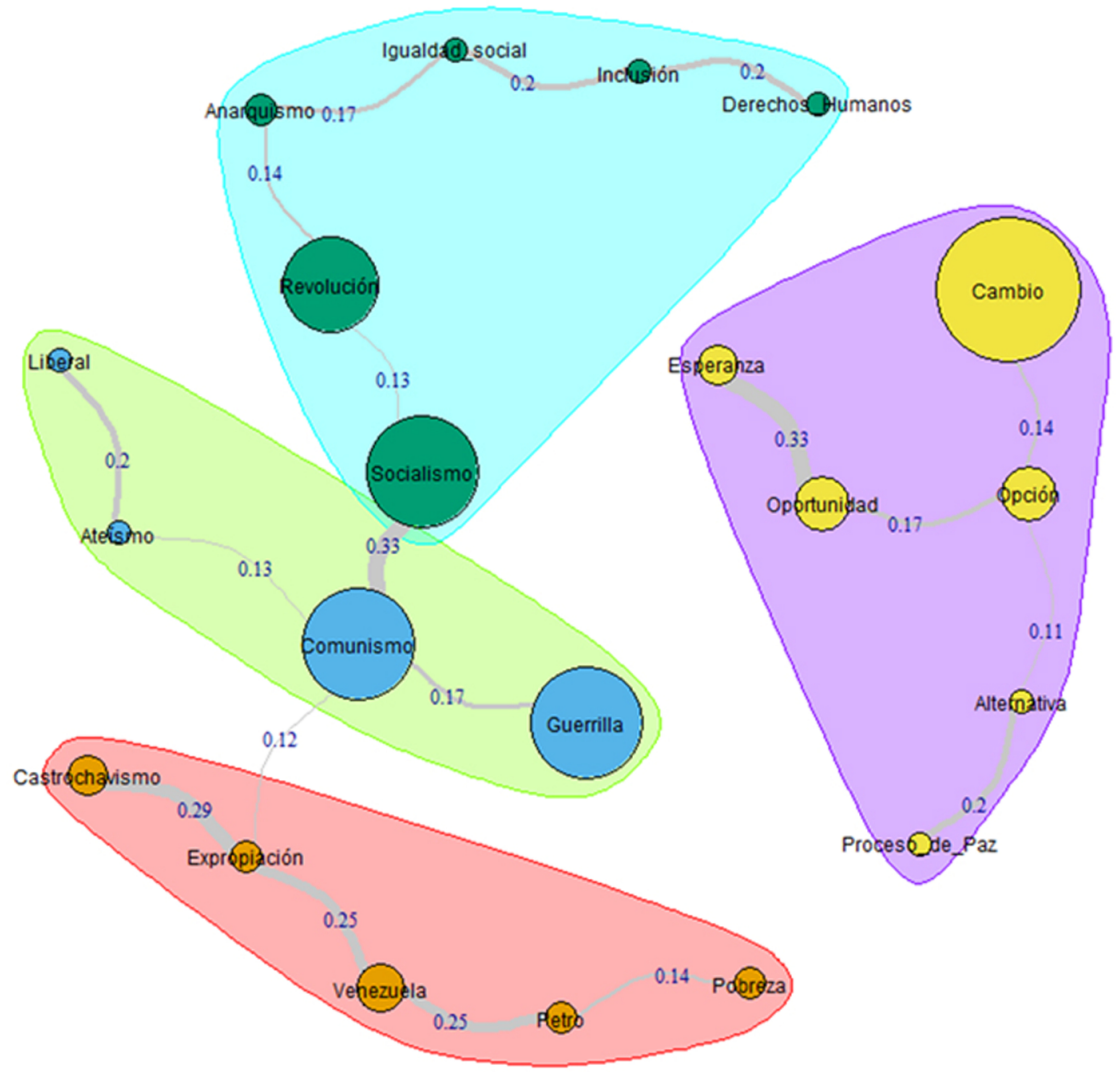

Figura 2. Análisis de similitud del término inductor 'izquierda' Fuente: elaboración propia.

Ahora bien, en lo que se refiere al efecto movilizador de la etiqueta asociada a la imagen de los candidatos Iván Duque y Gustavo Petro, no se evidenció significancia desde el punto de vista estadístico $(p<0,05)$ al comparar los posicionamientos de los votantes de derecha que evaluaron las premisas como si provinieran del programa de gobierno de Duque o del programa de gobierno de Petro; en otras palabras, la fotografía de los candidatos, que fue presentada de forma intencional, aparentemente no incidió en los posicionamientos de aprobación o desaprobación de las premisas por parte de este grupo.

Con los votantes de izquierda ocurrió algo diferente. Como muestran los resultados de la tabla 5, el ejercicio de comparación de medias evidenció diferencias significativas $(p<0,05)$ en cinco de las siete premisas, a saber, alimentación balanceada de los niños $[F(1,18)=7,58 ; p<0,013]$, investigación científica y tecnológica $[F(1,18)=17,05 ; p<0,001]$, reactivación del agro $[F(1,18)=12,38 ; p<0,002]$, equidad de género $[F(1,18)=4,93 ; p<0,039]$ y sostenibilidad ambiental $[F(1,18)=9,70 ; p<0,006]$, lo que se puede interpretar como un efecto movilizador del rótulo, es decir, parte de los 
electores de izquierda aprobaron las premisas cuando provenían del programa de Gustavo Petro y la otra parte las desaprobó o se posicionó de forma neutral porque correspondían al candidato Iván Duque.

Con respecto a los votantes que el estudio definió como de centro, solo hubo significancia en una de las siete premisas, relacionada con el estímulo del emprendimiento como estrategia que contribuye a la formalización del empleo $[F(1,58)=5,77$; $p<0,019]$, lo que quiere decir que en los demás postulados la etiqueta "partido de derecha" o "partido de izquierda" no surtió un efecto movilizador, como evidentemente ocurrió con los participantes que fueron definidos como votantes de izquierda para efectos del estudio.

Tabla 5. Comparación de las medias según Test hsd de Tukey

\begin{tabular}{|c|c|c|c|c|c|c|c|c|c|}
\hline \multirow{2}{*}{ Premisa/ Rótulo } & \multicolumn{2}{|c|}{$\begin{array}{l}\text { Votantes de } \\
\text { Izquierda }\end{array}$} & \multirow[t]{2}{*}{ Sig. } & \multicolumn{2}{|c|}{ Votantes de centro } & \multirow{2}{*}{ Sig. } & \multicolumn{2}{|c|}{$\begin{array}{l}\text { Votantes de } \\
\text { derecha }\end{array}$} & \multirow{2}{*}{ Sig. } \\
\hline & Petro & Duque & & Izquierda & Derecha & & Petro & Duque & \\
\hline $\begin{array}{l}\text { Alimentación } \\
\text { balanceada }\end{array}$ & 2,0 & 0,78 & $0,013^{*}$ & 1,89 & 1,82 & 0,575 & 1,70 & 2,00 & 0,121 \\
\hline $\begin{array}{l}\text { Jornada escolar } \\
\text { única }\end{array}$ & 1,09 & 0,11 & 0,096 & 1,12 & 0,76 & 2,208 & 1,10 & 1,08 & 0,956 \\
\hline $\begin{array}{l}\text { Investigación } \\
\text { científica }\end{array}$ & 1,82 & 0,44 & $0,001^{*}$ & 1,70 & 1,47 & 0,277 & 1,50 & 1,77 & 0,274 \\
\hline $\begin{array}{l}\text { Emprendimiento } \\
\text { para el empleo }\end{array}$ & 1,27 & 0,44 & 0,085 & 1,81 & 1,30 & $0,019^{*}$ & 1,30 & 1,50 & 0,442 \\
\hline $\begin{array}{l}\text { Reactivación del } \\
\text { campo }\end{array}$ & 1,91 & 0,22 & $0,002^{*}$ & 1,56 & 1,35 & 0,321 & 1,20 & 1,69 & 0,111 \\
\hline Equidad de género & 1,91 & 1,00 & $0,039^{*}$ & 1,67 & 1,47 & 0,324 & 0,80 & 1,38 & 0,248 \\
\hline $\begin{array}{l}\text { Sostenibilidad } \\
\text { ambiental }\end{array}$ & 2,00 & 1,22 & $0,006^{*}$ & 1,85 & 1,82 & 0,838 & 1,70 & 1,69 & 0,978 \\
\hline
\end{tabular}

*Premisas con significancia estadística.

Fuente: elaboración propia.

\section{Discusión}

Con el presente estudio nos propusimos identificar tanto la posible representación social de la derecha y la izquierda por parte del grupo de participantes como el efecto movilizador de la imagen cuando se trata de aprobación o rechazo de premisas provenientes de programas de gobierno que se enmarcan en esas corrientes ideológicas: Iván Duque, en el caso de la derecha y Gustavo Petro, en el caso de la izquierda en Colombia. 
Estamos refiriéndonos, sin duda, a dos temas conexos desde el punto de vista psicosocial, toda vez que una de las funciones de las representaciones sociales, entendidas como teorías ingenuas que se construyen en el marco del sentido común, es contribuir a la formación de la identidad social de un grupo, arrojando pistas para entender procesos como la categorización y la comparación social.

Con respecto a la representación socialmente construida de la derecha y la izquierda, es de suma importancia destacar dos aspectos que llaman la atención. El primero tiene que ver con la naturaleza de los elementos hallados en el análisis prototípico: tanto en la representación social de la derecha como en las dos posibles representaciones de la izquierda, es evidente el predominio de elementos normativos, asociados a juicios de valor, soportados en una perspectiva ideológica e, incluso histórica, en lugar de elementos funcionales, vinculados a las prácticas sociales, lo cual es comprensible, pues se trata de objetos que generan polémicas y posicionamientos en el marco del endogrupo y de las mismas relaciones intergrupales, especialmente, en momentos de movilización electoral.

Ahora bien, al cotejar el análisis prototípico con las relaciones entre cognemas que arrojó el análisis de similitud, aparece el segundo aspecto que vale la pena destacar, esta vez vinculado al pensamiento social como lo propone Rouquette (1998), es decir, como un efecto de campo, que no se agota en la designación de contenidos lexicales evocados, sino que se convierte en un sistema o "dispositivo de sociabilidad" (p. 41) en el que convergen: 1) la memoria compartida, 2) la diferenciación intergrupal marcada por la alteridad y 3) la diversidad de juicios individuales, como dimensiones interrelacionadas que forman campos semánticos o de significación atravesados por una lógica sociohistórica.

Cuando Melo (2017) hace alusión a culturas incompatibles que le daban un "aire de cruzada y guerra santa" (p. 146) a los partidos Liberal y Conservador en el siglo XIX y principios del XX en Colombia; o cuando Rehm (2014) menciona los "odios heredados" (p. 20) como elemento cultural relacionado con la pertenencia a esos partidos políticos, no se están refiriendo a interpretaciones congeladas de una historia distante, sino a vectores de la memoria que continúan dinamizando esos campos semánticos o de interpretación del mundo político contemporáneo en el país.

Dichos cuadros de interpretación de la realidad, que no son otra cosa que representaciones sociales de un objeto (la derecha o la izquierda), al ser construidos a partir de la lógica no metódica del sentido común y de un principio de analogía débil diferente del conocimiento que emana del formalismo científico-racional-, terminan siendo simplificaciones de la realidad, lo cual es precisamente una característica del pensamiento social. En el estudio realizado, esa simplificación de la realidad se hace 
evidente, por ejemplo, al observar la forma en que comunismo se asocia con ateísmo en una de las representaciones de la izquierda, argumento histórico que motivó la guerra entre conservadores y liberales radicales de la primera mitad del siglo XX; o la relación de ese cognema con la crisis política y económica de Venezuela, que ha tenido efectos directos en Colombia con la migración masiva de ciudadanos venezolanos que buscan mejores condiciones de vida.

Ese mismo vector de la memoria histórica aparece también en los campos semánticos relacionados con la representación social de la derecha, al asociar la figura del expresidente Álvaro Uribe con eventos que fueron escándalos mediáticos en Colombia, como los nexos del poder político con el paramilitarismo; o los falsos positivos, una estrategia de algunos miembros del Ejército Nacional de Colombia, que consistía en asesinar civiles y hacerlos pasar por guerrilleros muertos en combate, con el propósito de mostrar la efectividad en el marco de la política de "seguridad democrática", bandera del entonces mandatario nacional.

Este análisis nos lleva a encontrar un puente de conexión con la tesis de Albarracín (2020), según la cual el concepto de izquierda y derecha, presente en la opinión pública de países como Colombia, no hace alusión a ideas abstractas e interconectadas sobre cómo entender la política, sino que refleja más "un sentido de pertenencia y apego psicológico a un grupo social, en forma análoga a las identidades partidarias, que la adhesión a un conjunto de ideas" (p. 15). Dicho en otras palabras, aunque el pensamiento académico asocia la derecha política a un Estado menos intervencionista en términos económicos y la izquierda política a un Estado más intervencionista y orientado a reducir las desigualdades sociales, el pensamiento social no aprehende esos mismos conceptos, sino que los simplifica, adaptándolos a una lógica interaccional.

Ahora bien, con respecto a la aplicación de la escala Likert, que se proponía evidenciar si la aceptación o rechazo de una premisa por parte de un grupo podría tener una relación con la presencia o ausencia de la imagen de los respectivos candidatos, conviene explicar que estudios similares ya se han realizado en el campo del pensamiento social, llevando a autores como Rouquette (1994) y Wolter (2009) a teorizar la existencia de objetos movilizadores, que no activan aspectos cognitivos, sino afectivos, y que causan aceptación o rechazo automático, eliminando incluso las diferencias intergrupales.

En lo relacionado con el posible efecto que tuvo el rótulo, especialmente en los votantes de izquierda en los que la comparación de medias arrojó significancia, es importante tener en cuenta que este se puede asociar al vector afecto, entendido como una experiencia de tensión dialógica, que se hace particularmente evidente, como lo 
explica Marková (2006), en las dinámicas de conflicto intergrupal. Asimismo, el rótulo también puede estar vinculado a una representación social o teoría ingenua sobre un objeto que, para el caso del presente estudio fueron Iván Duque, como candidato de derecha, y Gustavo Petro, como candidato de izquierda. Dichas representaciones, además de incidir en los posicionamientos, también orientan el sentimiento de pertenencia y la forma en que el grupo se ve a sí mismo y al grupo opositor, a través de los procesos de comparación y categorización social.

En ese orden de ideas, el rótulo movilizador se podría definir, como un gatillo o elemento activador de la representación social construida sobre un objeto determinado, abarcando, simplificando e, incluso, tergiversando cualquier contenido que se le asocie. Cuando la rotulación activa la representación asociada a la identidad social de un grupo específico, los posicionamientos pueden ser incluso más recalcitrantes, toda vez que está en juego la defensa de la pertenencia grupal frente a la amenaza de un exogrupo, que tiene posicionamientos opuestos y una identidad social diferente.

Como demostraron Tajfel e Wilkes (1963), una rotulación impuesta puede afectar la forma en que una persona percibe o juzga su entorno, teniendo en cuenta que la categorización se activa inmediatamente. De acuerdo con los resultados del presente estudio fue posible evidenciar que los votantes de izquierda se mostraron fuertemente favorables a las premisas cuando estaban amparadas bajo el rótulo de Gustavo Petro, y neutros o contrarios cuando era Iván Duque quien las proponía; en otras palabras, más que el contenido en sí mismo de las premisas, lo que primó fue el efecto movilizador del rótulo Iván Duque y la representación socialmente construida, no necesariamente sobre él como persona, pues se trataba de una figura poco conocida en el ámbito político colombiano hasta antes de ser catapultado por el expresidente Álvaro Uribe, sino del grupo político de derecha que lo respaldaba.

Es importante destacar que las premisas no abordaban temas polémicos ni denotaban un sesgo político específico, pues se intentó presentar una redacción neutra, lo que a la postre permitió evidenciar el efecto del rótulo; ahora bien, si se hubiese tratado de premisas abiertamente polémicas, es probable que su efecto hubiese sido más evidente, como de hecho ocurre comúnmente en las redes sociales digitales.

\section{Conclusión}

Presentamos los resultados de un estudio realizado con 104 participantes durante la campaña presidencial de 2018 en Colombia, con el objetivo de identificar la posible representación social de los conceptos 'izquierda' y 'derecha', así como el efecto 
movilizador del rótulo o etiqueta asociado a la imagen de los candidatos Gustavo Petro e Iván Duque, adscritos a dichas corrientes políticas respectivamente.

Con respecto a la representación social de la derecha y la izquierda, fue posible identificar campos semánticos en los que aparece Álvaro Uribe como un elemento articulador de la representación de la derecha, que podríamos denominar monolítica, dada la aparente centralidad de la figura del expresidente en la estructura de relaciones encontrada, a diferencia de las dos posibles representaciones de la izquierda, una vinculada históricamente al comunismo y otra, a las expectativas de cambio generadas por el proceso de paz con las FARC.

Al analizar el efecto movilizador del rótulo fue posible evidenciar que este se comporta como activador de la representación social, especialmente, cuando se relaciona con la identidad social de un grupo, favoreciendo el estereotipo o simplificación de la realidad e incidiendo en los posicionamientos de sus integrantes con respecto a premisas contenidas en los respectivos programas de gobierno.

Teniendo en cuenta que el estudio realizado surgió a partir de la observación sistemática de una red social digital en momentos de - podríamos llamarlo así- paroxismo político en Colombia, consideramos fundamental presentar estos resultados como catalizadores de una reflexión académica y ciudadana, de modo que se propongan nuevas investigaciones, no solo en el campo de la Psicología Social, sino de las Ciencias Humanas y Sociales, toda vez que ese tipo de fenómenos, hoy tan frecuentes en escenarios electorales caracterizados por lo que algunos analistas políticos denominan polarización, no es ajeno a otros contextos políticos y se hace más evidente justamente en el campo de las redes sociales digitales, pues son plataformas de interacción donde se genera, circula y se reproduce el pensamiento social en la contemporaneidad.

Experiencias históricas de movilización a favor de una democracia real se han materializado a partir de las redes sociales digitales (Castells, 2013; Wolfsfeld et al., 2013); pero también se ha demostrado el poder distorsionador de las mismas en otros contextos, donde ha primado la divulgación automatizada de noticias falsas, verbigracia la campaña presidencial de los Estados Unidos en 2016 (Guess et al., 2018) o el Brexit en el Reino Unido (Polonski, 2016). No obstante los controles implementados por dichas plataformas, el foco de problematización sigue siendo el mismo: la comunicación humana mediada por dispositivos electrónicos y el papel que estos ejercen en la forma en que los grupos humanos piensan y toman decisiones que los afectan directamente.

Entender la lógica de formación del pensamiento social y la identidad social de los grupos permite contar con elementos de interpretación y análisis, que pueden 
servir de brújula para orientarnos en territorios que la lógica científico-racional describiría como confusos o difícilmente navegables, pero que son absolutamente actuales y transversales a cualquier ejercicio de educación para la(s) ciudadanía(s).

\section{Referencias}

Abric, J. C. (1994). Pratiques sociales et représentations. Presses Universitaires de France.

Albarracín, J. (2020). Explorando las bases: opinión pública y los sentidos de la derecha en Brasil y Colombia. En O. A. Bravo (Ed.), Las nuevas derechas: un desafío para las democracias actuales (pp.13-42). Editorial Icesi.

Alto Comisionado para la Paz de Colombia. (2016). Acuerdo general para la terminación del conflicto y la construcción de una paz estable y duradera. https://bit.ly/3zuyAYM

Álvaro, J. L. y Garrido, A. (2006). Psicologia social: perspectivas psicológicas e sociológicas. McGraw-Hill.

Amâncio, L. (2004). Identidade social e relações intergrupais. En J. Vala y M. B. Monteiro (Orgs.), Psicologia Social (pp. 387-400). Fundação Calouste Gulbenkian.

Basset, Y. (2018). Claves del rechazo del plebiscito para la paz en Colombia. Estudios Políticos, 52, 241-265. https://doi.org/10.17533/udea.espo.n52a12

Botero, S. (2017). El plebiscito y los desafíos de consolidar la paz negociada en Colombia. Revista de Ciencia Política, 37(2), 368-388. http://dx.doi.org/10.4067/s0718-090×2017000200369

Cabecinhas, R. (2007). Preto e branco: A naturalização da discriminação racial. Oporto, Portugal: Campo das Letras.

Cardona, L. M. y Londoño, C. A. (2018). La retórica del miedo como estrategia política: el plebiscito por la paz en Colombia. Forum, 14, 43-68. https://doi.org/10.15446/frdcp.n14.69614

Casey, N. (2016, 24 de agosto). Colombia and FARC Reach Deal to End the America's Longest War. New York Times. https://nyti.ms/3zw1t60

Castells, M. (2013). Redes de indignação e esperança: movimentos sociais na era da internet. Zahar. 
Pensamiento social y posicionamiento político: representaciones sociales de la derecha y la izquierda en Colombia

Castorina, A. (2016). El significado del marco epistémico en la teoría de las representaciones sociales. Cultura y representaciones sociales, 11(21), 79-108.

Centro Nacional de Memoria Histórica de Colombia (CNMH). (2013). jBasta ya! Memorias de guerra y dignidad: Informe general Grupo de Memoria Histórica. https://bit.ly/3gNUy1r

Dancey, C. y Reidy, J. (2006). Estatística sem matemática para a psicologia: Usando SPSS para Windows. Artmed.

Deschamps, J-C. (1983). Identidade e categorização social. Psicologia, 4, 123-138.

Deschamps, J-C. y Moliner, P. (2009). A identidade em psicologia social: Dos processos identitários às representações sociais. Editora Vozes.

Doise, W. (1987). Le social et l'individuel: Théories générales et recherches intergroupes. Psychologie et Éducation, 11, 57-74.

Doise, W. (1988). Individual and Social Identities in Intergroup Relations. European Journal of Social Psychology, 18, 99-111.

Echeverri, G. y Andrade, R. (2019). Redes sociais digitais: a lógica do pensamento social em eventos de mobilização coletiva.Polêmica, 19(2), 1-17. https://doi.org/10.12957/polemica.2019.46672

Echeverri, G., Wolter, R. P.y Peixoto, Á. (2019). Redes sociales digitales y pensamiento social: el caso de la ocupación de las escuelas de Río de Janeiro. Psicología, Conocimiento y Sociedad, 9(1), 106-131. http://dx.doi.org/10.26864/pcs.v9.n1.1

Fiske, S. (1998). Stereotyping, Prejudice, and Discrimination. En D. Gilbert, S. Fiske y G. Lindzey (Eds.), The Handbook of Social Psychology (pp. 357-411). McGraw-Hill.

Gaymard, V. (2016, 24 de agosto). Colombie: la signature des accords de paix, une journée historique. Radio France International. https://bit.ly/3mLKhqh

Guess, A., Nyham, B. y Reifler, J. (2018). Selective Exposure to Misinformation: Evidence from the Consumption of Fake News During the 2016 U.S. Presidential Campaign. European Research Council. https://bit.ly/3jwA2Et

Gómez, R. M. (2017). El plebiscito sobre la paz en Colombia: la legítima búsqueda de la paz en un contexto antagónico. Revista de Derecho y Ciencias Sociales, 13, 265-278. 
Jodelet, D. (1989). Représentations sociales: un domaine en expansion. En D. Jodelet (Ed.), Les Représentations Sociales (pp. 45-78). Presses Universitaires de France.

Marková, I. (2006). Dialogicidade e representações sociais: a dinâmica da mente, tradução de Hélio Magri Filho. Vozes.

Marques, J. (1988). Categorização social, identidade social e homogeneidade de outgroup: uma análise conceptual. Análise Psicológica, 3-4(6), 279-305.

Marques, J. y Páez, D. (2004). Processos cognitivos e estereótipos sociais. En J. Vala y M. B. Monteiro (Eds), Psicologia Social (pp. 333-384). Fundação Calouste Gulbenkian.

Melo, J. O. (2017). Historia mínima de Colombia. Turner Publicaciones.

Moscovici, S. (1961). La psychanalyse, son image et son public. Presses Universitaires de France.

Oliveira, D. C., Marques, S.C., Gomes, A. M. T, y Teixeira, M. C. T. V. (2005). Análise das Evocações Livres: uma técnica de análise estrutural das representações sociais. En A. S. P. Moreira, B. V. Camargo, J. C. Jesuíno, y S. M. Nóbrega (Orgs.), Perspectivas Teórico-Metodológicas em Representações Sociais (pp. 573-603). Editora Universitária da Universidade Federal da Paraíba.

Pechenkina, A. y Gamboa, L. (2019). Who Undermines the Peace at the Ballot Box? The case of Colombia. Terrorism and Political Violence. https://doi.org/10.1080/09546553.2019.1676239

Polonski, V. (2016). Impact of social media on the outcome of the EU referendum. Blog Views from Oxford. https://bit.ly/3yuzgvQ

Real, R., y Vargas, M. (1996). The Probabilistic Basis of Jaccard's Index of Similarity. Systematic Biology, 45(3), 380-385. https://doi.org/10.1093/sysbio/45.3.380

Rhem, L. (2014). La construcción de las subculturas políticas en Colombia: los partidos políticos como antípodas políticas durante La Violencia, 1946-1964. Historia y Sociedad, 27, 17-48. como antípodas políticas durante La Violencia, 1946-1964. Historia y Sociedad, 27, 17-48. https://doi.org/10.15446/hys.n27.44582

Rouquette, M.-L. (1994). Sur la connaissance des masses: essai de psychologie politique. Presses Universitaires de Grenoble.

Rouquette, M.-L. (1998). La communication sociale. Dunod. 
Pensamiento social y posicionamiento político: representaciones sociales de la derecha y la izquierda en Colombia

Sá, C. P. (1998). A construção do objeto de pesquisa em representações sociais. Vozes.

Sá. C.P. de (2015). Estudos de Psicologia Social: história, comportamento, representações e memória. EdUERJ.

Tajfel, H. y Turner, J.C. (1986). The Social Identity Theory of Intergroup Behavior. En S. Wolchel y W. G. Austin (Orgs.), Psychology of Intergroup Relations (pp. 7-24). Nelson Hall.

Tajfel, H. (1981). Grupos humanos e categorias sociais. Lisboa, Portugal: Livros Horizonte.

Tajfel, H. (1978). Differenciation Between Social Groups: Studies in the Social Psychology of Intergroup Relations. Academic Press.

Tajfel, H., y Wilkes, A. (1963). Classification and Quantitative Judgement. British Journal of Psychology, 54, 101-114.

Uribe-Mallarino, C. (2008). Estratificación social en Bogotá: de la política pública a la dinámica de la segregación social. Universitas Humanística, 65, 139-171. https://bit.ly/3gLMCxC

Vergès, P. (1994) Approche du noyau central: propriétés quantitatives et structurales. En C. Guimelli, Structures ettransformations des représentations sociales (pp. 233-253). Delachaux et Niestlé.

Wachelke, J. y Wolter, R. (2011). Critérios de construção e relato da análise prototípica para representações sociais. Psicologia: Teoria e Pesquisa, 27(4), 521-526. https://doi.org/10.1590/ S0102-37722011000400017

Wolfsfeld, G., Segev, E. y Sheafer, T. (2013). Social Media and the Arab Spring: Politics Comes First. The International Journal of Press/Politics, 18(2), 115-137. https://doi.org/10.1177 /1940161212471716

Wolter, R. P. (2009). Les objets à forte valence affective : la notion de nexus. En M.-L. Rouquette (Org.), La pensée sociale: perspectives fondamentales et recherches appliquées (pp. 59-72). Éditions Érès.

Wolter, R. P. (2018). The Structural Approach to Social Representations: Bridges between Theory and Methods. Psico-USF, 23(4), 621-631. https://doi.org/10.1590/1413-82712018230403 